Abstract 147 Table 1

\begin{tabular}{llll}
\hline & Preceding & Hypopnoea & Recovery \\
\hline$\left.\mathrm{LB}\right|^{*}$ & $1.4(0.1)$ & $1.3(0.1)$ & $1.3(0.1)$ \\
$\mathrm{RR} / \mathrm{min}^{*}$ & $70(11)$ & $13(7)$ & $68(24)$ \\
$\Theta\left(^{\circ}\right)$ & $65 \pm 37$ & - & $54 \pm 36$ \\
$\Delta \mathrm{EELV}(\mathrm{VT}$ units) \# & 0 & $-0.3(-1.1,0.5)$ & $-0.2(-1.1,2.2)$ \\
$\Delta \mathrm{VT}(\mathrm{VT}$ units)\# & 1 & $0.2(0.3)$ & $1(0.6)$ \\
Co-efficient of variation (CV) for VT $(\%)^{*}$ & $40(31)$ & $65(41)$ & $38(24)$ \\
\hline
\end{tabular}

*mean (SD) \#median (range)

Conclusions A significant loss in EELV may occur during hypopneic phases of respiration in infants considered stable on CPAP. Further work is needed to determine the significance of these observations.

\section{PARTIAL LUNG AERATION CAUSES VENTILATION/ PERFUSION MISMATCH IN THE LUNGS AT BIRTH}

doi:10.1136/archdischild-2012-302724.0148

'SB Hooper, ${ }^{1} \mathrm{~J}$ Lang, ${ }^{2} \mathrm{JT}$ Pearson, ${ }^{1} \mathrm{MJ}$ Wallace, ${ }^{1} \mathrm{ML}$ Siew, ${ }^{3} \mathrm{MJ}$ Kitchen, ${ }^{4} \mathrm{~A}$ Fouras, ${ }^{5} \mathrm{~K}$ Wheeler, ${ }^{1} \mathrm{GJ}$ Polglase, ${ }^{6} \mathrm{AB}$ te Pas. ${ }^{\prime}$ Monash Institute for Medical Research; ${ }^{2}$ Monash Biomedical Imaging; ${ }^{3}$ School of Physics; ${ }^{4}$ Division of Mechanical and Aerospace

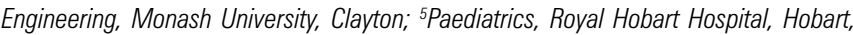
VIC, Australia; ${ }^{6}$ Pediatrics, Leiden University Medical Center, Leiden, The Netherlands

Background Although lung aeration increases pulmonary blood flow $(\mathrm{PBF})$ at birth, the regional relationships between lung aeration and the increase PBF are unknown. We investigated the effect of partial ventilation on pulmonary vessels immediately after birth using simultaneous phase contrast X-ray imaging and angiography. Method Newborn rabbits were delivered near-term ( $~ 30 \mathrm{~d}$ GA; term $\sim 32 \mathrm{~d}$ GA) and an iodine contrast agent was infused into the jugular vein before and then during both unilateral (of the right lung) and then ventilation of both lungs. Visible vessels were counted and diameters and integrated intensity line profiles that transected vessels at different locations were measured; the latter provides a relative measure of $\mathrm{PBF}$.

Results Unilateral ventilation of the right lung increased visible vessel number (from $15 \pm 1$ to $44 \pm 4$ ), vessel diameter (from $493 \pm 80 \mu \mathrm{m}$ to $543.2 \pm 84.3 \mu \mathrm{m}$ ) and integrated intensity (from $2496 \pm 472 \mu \mathrm{m}$.AU to $6594 \pm 658 \mu \mathrm{m} . \mathrm{AU}$ ) in the left lung while it was still liquid-filled and unaerated. As a result, the visible vessel number of perfused pulmonary vessels (right: $42 \pm 4$, left: $44 \pm 4$ ), mean vessel diameters and integrated intensity (left: $6594 \pm 658 \mu \mathrm{m}$.AU, right: $8012 \pm 1423$ $\mu \mathrm{m} . \mathrm{AU})$ were not different between aerated and non-aerated lung regions. Angiography videos demonstrating the spatial and temporal changes in PBF after birth will also be presented.

Conclusion Partial lung aeration promotes a global increase in PBF resulting in a highly significant ventilation/perfusion mismatch in unventilated lung regions. These observations indicate that a previously unsuspected mechanism contributes to the increase in PBF at birth.

\section{EVALUATION OF NEEDLE ACUPUNCTURE ACTIONS ON CLINICAL IMPROVEMENTPULMONARY FUNCTIONS, INTERLEUKIN-6, IMMUNOGLOBULIN-E AND MEDICATIONS USED OF ASTHMATIC CHILDREN}

doi:10.1136/archdischild-2012-302724.0149

'NH Mohamed, ${ }^{2} \mathrm{AEE}$ Abdel Meguid, ${ }^{2} \mathrm{HH}$ Shaaban, 'IEM Ahmed Kamel. 'Researches and Applications of Complementary Medicine Department, National Research Center; ${ }^{2}$ Pediatric Department, Cairo University, Cairo, Egypt

Background and aims Bronchial asthma is one of the most common illnesses in children. The chronicity of bronchial asthma and the fear of steroid therapy cause many patients to seek alternative methods of treatment such as Acupuncture, herbal medicine and massage therapy. The complementary and alternative medicine is a widespread phenomenon, In Europe, complementary therapies are used by $20-50 \%$ of the population. Among pediatric populations, Acupuncture is considered the backbone of the CAM modalities.

Methods we applied the acupuncture sessions for 30 cases of children who suffering from asthma on their conventional medical treatment on certain points according to Traditional Chinese Medicine for 12 sessions in one month and measuring of Pulmonary functions including (VC\%, FVC\%, FEV1\%, FEF25-75\%, FEF25\%, FEF50\%, FEF75\%, PEF\%), IL-6, IgE, Eosinophilic count before and after the sessions. Clinical grading and medications used before and after the acupuncture sessions measured.

Results There were significant improvement in Pulmonary functions: VC $\%(p<0.001)$, FVC $\%(p<0.001)$, FEV1\% $(p<0.001)$ FEF25-75\% $\quad(p<0.001), \quad$ PEF\% $\quad(p<0.001), \quad$ FEF25\% $\quad(p<0.001)$ FEF50\% $(p<0.001)$, FEF $75 \%(p<0.001)$. There were significant decrease in Eosinophilic count $(p<0.001)$. More over there were improvement in IL- 6 and IgE levels in the blood. However there were improvements in clinical conditions of the patients (symptoms, signs, grade of asthma) also there was decrease in their medications used

Conclusions Acupuncture improves pulmonary functions of the children with bronchial asthma however it decreases the serum levels of Eosinophilic count, IL-6 and IgE with decrease all medications used by children before needle sessions.

\section{NEUROCOGNITIVE DEVELOPMENT OF CHILDREN FOUR YEARS AFTER CRITICAL ILLNESS AND TREATMENT WITH TIGHT GLUCOSE CONTROL: A RANDOMIZED, CONTROLLED TRIAL}

doi:10.1136/archdischild-2012-302724.0150

${ }^{1} \mathrm{M}$ Gielen, 'D Mesotten, 'C Sterken, 'K Claessens, 'G Hermans, 'D Vlasselaers, ${ }^{2} \mathrm{~J}$ Lemiere, ${ }^{3} \mathrm{~L}$ Lagae, ${ }^{4} \mathrm{M}$ Gewillig, ${ }^{4} \mathrm{~B}$ Eyskens, II Vanhorebeek, ${ }^{1} \mathrm{PJ}$ Wouters, ${ }^{1} \mathrm{G}$ Van den Berghe. 'Intensive Care Medicine; ${ }^{2}$ Child and Adolescent Psychiatry; ${ }^{3}$ Pediatric Neurology; ${ }^{4}$ Pediatric and Congenital Cardiology, KULeuven, Leuven, Belgium

Background and aims The first large RCT on tight-glucosecontrol (TGC) to age-adjusted normoglycemia in the pediatricintensive-care-unit (PICU) (Vlasselaers 2009) revealed that TGC reduced PICU morbidity and mortality as compared with usualcare, but increased hypoglycemia $\leq 40 \mathrm{mg} / \mathrm{dL}$. As both hyper- and hypoglycemia may adversely affect the developing brain, an assessment of long-term neurocognitive function was required to exclude harm and validate any short-term benefit of TGC.

Methods Follow-up of all 700 patients included in the original RCT, was performed 4 years after randomization. Death or disability precluding neurocognitive testing were a priori defined as poor outcomes. The primary endpoint was full-scale IQ, assessed with age-adjusted intelligence-tests (Wechsler-IQ-scales). Neurodevelopmental-testing also encompassed a neurological examination, and tests for visual-motorintegration (VMI-Beery-Buktenica-Developmental-Test), attention and executive functions (ANT-Amsterdam-Neuropsychological-Tasks), memory (Children's-Memory-Scale), and behavior (Child-BehaviorCheckList). For comparison, 216 healthy siblings and unrelated children were tested.

Results At follow-up, TGC in PICU had not increased the incidence of poor outcomes $[19 \%$ vs. $18 \%$, univariable OR for poor outcome with TGC $1.10(0.76-1.62), P=0.6]$. Sixteen percent of the 700 ICU patients declined participation or were not contactable. TGC did not affect full-scale IQ [median 88.0 (IOR 74.0-100.0) vs. 88.5 (74.3-99.0), $P=0.7]$, nor other scores for intelligence, visual-motor-integration, memory and behavior. TGC actually improved motor coordination (all $P \leq 0.03$ ) and cognitive flexibility $(P=0.02)$, the latter up to the level of healthy children. 
Imputation for missing values confirmed these results. Hypoglycemia evoked by TGC did not negatively affect neurocognitive outcome.

Conclusion Despite hypoglycemia, TGC in PICU did not harm neurocognitive development 4 years later.

\section{CARDIAC FUNCTION AT THE AGE OF 7 YEARS OF REGIONAL BIRTH COHORT OF EXTREMELY LOW BIRTH WEIGHT INFANTS $(<$ 1000G)}

doi:10.1136/archdischild-2012-302724.0151

P Kwinta, M Jagla, A Grudzien, M Klimek, M Zasada, JJ Pietrzyk. Department of Pediatrics, Jagiellonian University, Cracow, Poland

Aim Assessment of long-term cardiac complications in the regional cohort of extremely low birth weight (ELBW) children born in 2002-2004.

Material and Methods The study group comprising 81 children born as ELBW infants with the median birthweight of $890 \mathrm{~g}\left(25^{\text {th }}\right.$ $75^{\text {th }}$ percentile: $760-950$ ) were evaluated at the mean age of 7 years. The control group included 40 children born full term. Echocardiography and 24-hour ambulatory blood pressure measurements were performed.

Results

Abstract 151 Table $1 \quad$ M-mode variables (presented as z-score)

\begin{tabular}{llll}
\hline & ELBW & Control & p \\
\hline RVDd & $-1.49 \pm 1,25$ & $-0.31 \pm 0,91$ & $<0.001$ \\
LVIDd & $-0.53 \pm 1,26$ & $0.13 \pm 0,94$ & 0.01 \\
Ao & $0.24 \pm 1,23$ & $0.83 \pm 1,03$ & 0.02 \\
LA & $-0.93 \pm 1,07$ & $-0.15 \pm 1,02$ & $<0.01$ \\
\hline
\end{tabular}

Abstract 151 Table 2 Results of 24-hour blood pressure monitoring

\begin{tabular}{llll}
\hline & ELBW & Control & p \\
\hline 24-h mean MAP(mmHg) & $79 \pm 5.9$ & $77 \pm 4.4$ & 0.2 \\
Systolic BP load(\%) & $28 \pm 22$ & $16 \pm 14$ & $<0.01$ \\
Diastolic BP load(\%) & $27 \pm 20$ & $17 \pm 10$ & $<0.01$ \\
Mean HR(bpm) & $93 \pm 8$ & $87 \pm 7$ & $<0.001$ \\
\hline
\end{tabular}

Abstract 151 Table 3 LVM, LVMI and cardiac index

\begin{tabular}{llll}
\hline & ELBW & Control & p \\
\hline LVM(g) & $48.8 \pm 15,5$ & $65.1 \pm 15,4$ & $<0.001$ \\
LVMI(g/m2.7) & $32.4 \pm 9,2$ & $35.7 \pm 8,3$ & 0.08 \\
Stroke index(/BSA) & $41.8 \pm 15,3$ & $47.9 \pm 12,5$ & 0,04 \\
Cl (I/m2) & $3.6 \pm 1,2$ & $3.8 \pm 1,04$ & 0.49 \\
\hline
\end{tabular}

Conclusions The former ELBW children have smaller heart's diameters and to reach the same cardiac index their heart rate is faster. Moreover, the former ELBW children have higher blood pressure comparing to their peers.

\section{PREDICTIVE FACTORS FOR SURVIVAL AFTER PAEDIATRIC OUT-OF-HOSPITAL CARDIAC ARREST: A UK MULTICENTRE COHORT STUDY}

doi:10.1136/archdischild-2012-302724.0152

1,2B Scholefield, ${ }^{3} \mathrm{~S}$ Raman, ${ }^{3} \mathrm{~A}$ Hussey, ${ }^{4} \mathrm{~F}$ Haigh, ${ }^{1} \mathrm{HK}$ Kanthimathinathan, ${ }^{3} \mathrm{~S}$ Skellett, ${ }^{3} \mathrm{M}$ Peters, 'H Duncan, 'K Morris. 'Paediatric Intensive Care Unit, Birmingham Children's Hospital, Birmingham; ${ }^{2}$ Medical School, University of Warwick, Warwick; ${ }^{3}$ Paediatric Intensive Care Unit, Great Ormond Street Hospital, London; ${ }^{4}$ Paediatric Intensive Care Unit, Alder Hey Childrens' Hospital, Liverpool, UK
Introduction Survival after paediatric out-of-hospital cardiac arrest (OHCA) is poor, even amongst those who are successfully resuscitated and admitted to PICU. Better prediction of survival would be of benefit to clinical teams and to research teams designing trials. This study aimed to identify predictive factors for survival to inform the design of a post-OHCA intervention trial.

Method Retrospective, cohort study of 155 infants and children ( 1 day to 16 years) admitted to 3 UK PICUs after OHCA (20042010). Variables relating to a) the resuscitation period (Utstein) and b) the post-resuscitation period were included in two multivariate stepwise regression models to identify predictive factors for survival to PICU discharge.

Results $32 \%$ (50/155) children survived to PICU discharge. Resuscitation variables individually associated with improved survival included; presenting in a shockable rhythm, shorter duration of arrest, return of spontaneous cardiac output prior to arrival in the emergency department and lower number of epinephrine doses. Post-resuscitation variables individually associated with improved survival included; higher arterial $\mathrm{pH}$, lower blood lactate, lower maximum glucose, higher base excess and responsive pupils. Results of multivariate stepwise regression models are reported in table.

Conclusion This large UK study is the first to identify lactate as one of the key predictors of paediatric OHCA survival in patients admitted to PICU. Development of an accurate prediction tool would assist trial design and prognostication after paediatric OHCA.

\begin{tabular}{|c|c|c|c|}
\hline Variable & $\begin{array}{l}\text { Odds Ratio } \\
\text { for survival }\end{array}$ & $95 \% \mathrm{Cl}$ & $P$ value \\
\hline \multicolumn{4}{|l|}{ Model 1: Resuscitation factors } \\
\hline $\begin{array}{l}\text { No epinephrine (versus one or more } \\
\text { doses of epinephrine during resuscitation) }\end{array}$ & 11.98 & 2.31 .61 .99 & 0.003 \\
\hline VFNT (versus PEA/asystolebradycardia) & 3.95 & $0.96-16.27$ & 0.057 \\
\hline \multicolumn{4}{|c|}{ (Model 1 : Area under receiver operating cunve $=0.67$ ) } \\
\hline \multicolumn{4}{|l|}{ Model 2: Post-resuscitation factors } \\
\hline Two unresponsive pupils (versus reactive) & 0,19 & $0.07-0.52$ & 0.001 \\
\hline Blood Lactate (per 1.0 mmoll increment) & 0.85 & $0.75-0.87$ & 0.015 \\
\hline \multicolumn{4}{|c|}{ (Model 2: Ales under receiver operating curve $=0.83$ ) } \\
\hline
\end{tabular}

\begin{tabular}{l}
\hline 153 VISUOSPATIAL AND EXECUTIVE FUNCTION IN \\
ADOLESCENT PATIENTS WITH CONGENITAL HEART \\
DISEASE
\end{tabular}

doi:10.1136/archdischild-2012-302724.0153

1,2M von Rhein, ${ }^{2} \mathrm{M}$ Kugler, ${ }^{2 B}$ Latal. 'Developmental Pediatrics, Kantonsspital, Winterthur; ${ }^{2}$ Child Development Center, University Children's Hospital, Zurich, Switzerland

Background and aims Visuospatial deficits have been described for 8 year-old patients with congenital heart disease (CHD) after bypass surgery based on the performance in the Rey-Osterrieth complex figure test (ROCFT). It is unknown whether these deficits persist into adolescence and which scoring systems is optimal to score performance in the ROCFT. We therefore performed ROCFT in adolescent CHD patients and healthy controls, and compared performance according to three different scoring methods.

Methods We examined 53 adolescents (mean age 13.7 years, 44\% male, $50 \%$ cyanotic CHD) with $\mathrm{CHD}$ after open-heart surgery 